\title{
Direct-to-consumer communication on prescription only medicines via the Internet in the Netherlands, a pilot study Opinion of the pharmaceutical industry, patient associations and support groups
}

\author{
- A. Mariette Fabius, Ka-Chun Cheung, Cristianne J.F. Rijcken, Christiaan H. \\ Vinkers and Herre Talsma
}

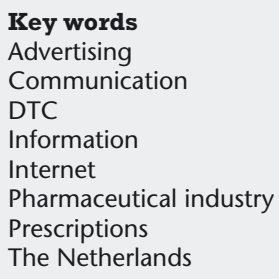

\section{Introduction}

Physicians, pharmacists and other health care providers receive promotional information on prescription medicines from pharmaceutical industries. Traditional channels are medical journals, personal contacts and post graduation courses. Nowadays, driven by growing patient interest in their own health affairs, a direct approach to the consumer is developing. This type of communication exists of information and advertising. In the USA, the pharmaceutical industry spent $\$ 2.6$ billion on direct-to-consumer (DTC) communication in 2000 . This amount is expected to rise to $\$ 7.5$ billion in $2005^{1}$. In Europe, DTC advertising is forbidden by law, although providing information directly to consumers is allowed. Only recently the European Commission proposed that pharmaceutical companies should be allowed to use DTC information in three chronic disease areas: HIV/AIDS, asthma and diabetes ${ }^{2,3}$.

DTC information has an objective and educational value, while a general purpose of advertising is to induce consumers to request prescriptions from their physicians ${ }^{4}$. Patients can use information to extend their knowledge about medicines, diseases and further therapeutic possibilities. In Europe, the distinction between information and advertisement in DTC communication for prescription only medicines is subtle and sometimes arbitrary.

Proponents of DTC communication emphasize the need for 'patient empowerment', as a patient has the right to receive accurate and reliable information. The pharmaceutical industry is willing to provide this information to the consumer ${ }^{5}$. Opponents state that a patient without a medical background is not always capable of understanding the offered content ${ }^{6}$. Furthermore, DTC information supplied via the internet sometimes lacks objectivity and reliability. In the Netherlands, an on-going self-regulation of pharmaceutical industry was suggested recently in order to minimize the chance of consumers being misled by DTC communication (C. de Visser, personal communication [2001]).

The possibility of gaining information on prescription medicines is highly appreciated by patients ${ }^{7}$. The relationship between patient and doctor might be affected by DTC communication in various, yet unclear aspects. A 1999 poll indicated that $91 \%$ of physicians felt pressurized by their patients ${ }^{8}$. Polls in the same year indicated that physicians welcome patient questions and respect their autonomy ${ }^{7}$. However, in another study from 2001, consumers still considered the physician as the primary source for information on prescription medicines ${ }^{9}$.

Increased discussions in Europe about DTC communication legislation, the role of the pharmaceutical industry and the growing autonomy of the patient all lead to the need of a better insight into DTC communication. Another simultaneous development in Europe is the fast rise of the Internet. This medium crosses all boundaries offering a huge amount of medical information. The majority of European citizens have access to the Internet and have the possibility to search for medical information. Most of the pharmaceutical companies have websites, thereby creating new DTC opportunities. The purpose of this pilot study was to present an overview of the current application and impact of DTC communication on prescription only medicines via the Internet in the Netherlands. Therefore, Dutch pharmaceutical companies and patients association and support groups (PASGs) in the Netherlands were asked to give their opinions on DTC communication via the Internet.

\section{Methods}

Questionnaires were used to investigate the current application and impact of DTC communication. In cooperation with two experts in the field of social sciences and pharmacoeconomics, two questionnaires 
were constructed. The questionnaires consisted of multiple choice questions (yes/no boxes and Likert scale boxes), questions with multiple answer possibilities, and open text questions for clarification.

In this pilot study the questionnaires were deliberately sent via e-mail in order to investigate the communication and response via e-mail. Questionnaires were sent to the innovative pharmaceutical industries which are member of Nefarma (Dutch Federation of Innovative Pharmaceutical Industries) and to patient association and support groups (PASGs) which are member of the master-federation Dutch Patients and Consumer Federation (NPCF). The e-mail addresses of the pharmaceutical industry were obtained by telephone. The e-mail addresses of PASGs were obtained via the website of the NPCF and were selected on diseases with a need of prescription only medicines. Assumed was that PASGs form a representation of the general opinion of patients in general or have sufficient knowledge of their members.

The questionnaire for the pharmaceutical industries concerned questions on creating and offering DTC communication via websites and website-related activities:

- website specificities

- activity on DTC communication

- contact with patients

The questionnaire for the PASGs concentrated on the visiting, using and judging the DTC possibilities as offered by the Dutch innovative pharmaceutical industry:

- website specificities

- attitude and opinion towards DTC communication

- patient-doctor relationship.

The adressed were asked to give their opinion as representative of the pharmaceutical industry or the opinion as representative of the PASGs. For PASGs, if this opinion was not known, the addressed could give their own opinion.

Both questionnaires were sent in June 2001 as a hyperlink in an e-mail to 43 innovative pharmaceutical industries and 130 PASGs. Two reminder e-mails were sent after two and four months to non-responders.

\section{Results}

\section{Pharmaceutical industry}

From the pharmaceutical industry, a total of $8 \mathrm{com}-$ pleted questionnaires were received (19\%). Separately, 10 e-mails (23\%) were received with various reasons for not participating in the questionnaires, among these were lacking a Dutch website, other priorities and rejection.

\section{Website specificities}

All responders had their own websites. The websites were maintained and controlled in different ways; four companies performed weekly updates, while five industries counted the number of visitors.

Printed advertisement was the main medium by which industries brought their websites to the notice of patients and health care providers. Two companies used folders at the physician's office. Sometimes a website was not promoted at all. Four industries linked to various websites, e.g., PASGs, other pharmaceutical could not indicate whether their websites were linked. Three companies registered web addresses with names of their products.

Activity on DTC communication

The intended receivers of information were medical professionals. They were offered information about the company, their products and relating diseases. Six companies used direct contact with patients to create a positive image. Other DTC-objectives included postmarketing surveillance, creating sales possibilities and providing information about medicines.

Five industries indicated that visitors regarded their information as reliable. Six companies believed that the direct contact with consumers influences marketing strategies. The cost-effectiveness balance of information on the Internet in comparison to other media was appreciated differently. Three industries recently raised the budget for Internet/email; the budget of three other companies remained unchanged.

\section{Contact with patients}

Five pharmaceutical companies enabled their visitors to ask on-line questions, subjects ranging from side effects, application, indication, expiring dates and reimbursement. Companies received up to 150 questions per month, and, in general, answered within a few days. Only one company supplied a monthly newsletter by e-mail to patients and medical professionals on request.

\section{Patient associations and support groups}

From the patient groups, a total of 46 completed questionnaires were received (35\%). Separately 20 emails (17\%) were received with various reasons for not participating in the questionnaires. These varied from problems with the website-server of the questionnaire, requests for more information, the lack of a financial compensation to no interest.

\section{Website specificities}

Most PASGs had their own website (89\% of responders). Of these websites $76 \%$ linked to various other information sources, like other (international) PASGs or related organizations. The majority did not link to websites of pharmaceutical companies (93\%). PASGs indicated that they generally do not obtain financial support from the pharmaceutical industry for Internet activities; only $11 \%$ was supported.

\section{Attitude towards DTC communication}

The majority (65\%) had no opinion whether the pharmaceutical industry provides a good distinction between advertising and information on their websites (Figure 1A).

The general DTC information via the Internet by the pharmaceutical industry was considered as reasonably positive (24\%) to neutral (17\%). However, $17 \%$ had no opinion and $30 \%$ did not answer the question (Figure 1B).

This 'no-opinion' attitude was also found in the answers about the impact of DTC communication. Of the respondents $50 \%$ had no opinion on the availability of drug innovations by websites, $24 \%$ expected that information on new innovations might become earlier available than before to patients, while $26 \%$ indicated no influence. 


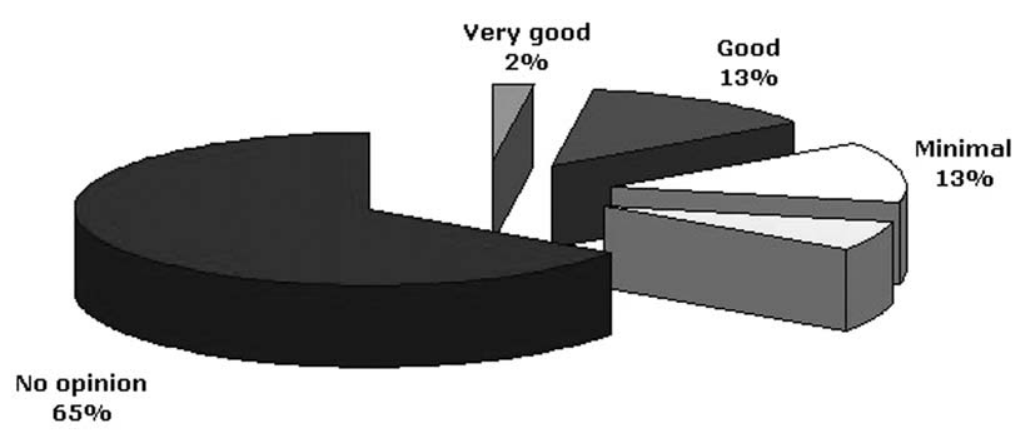

A
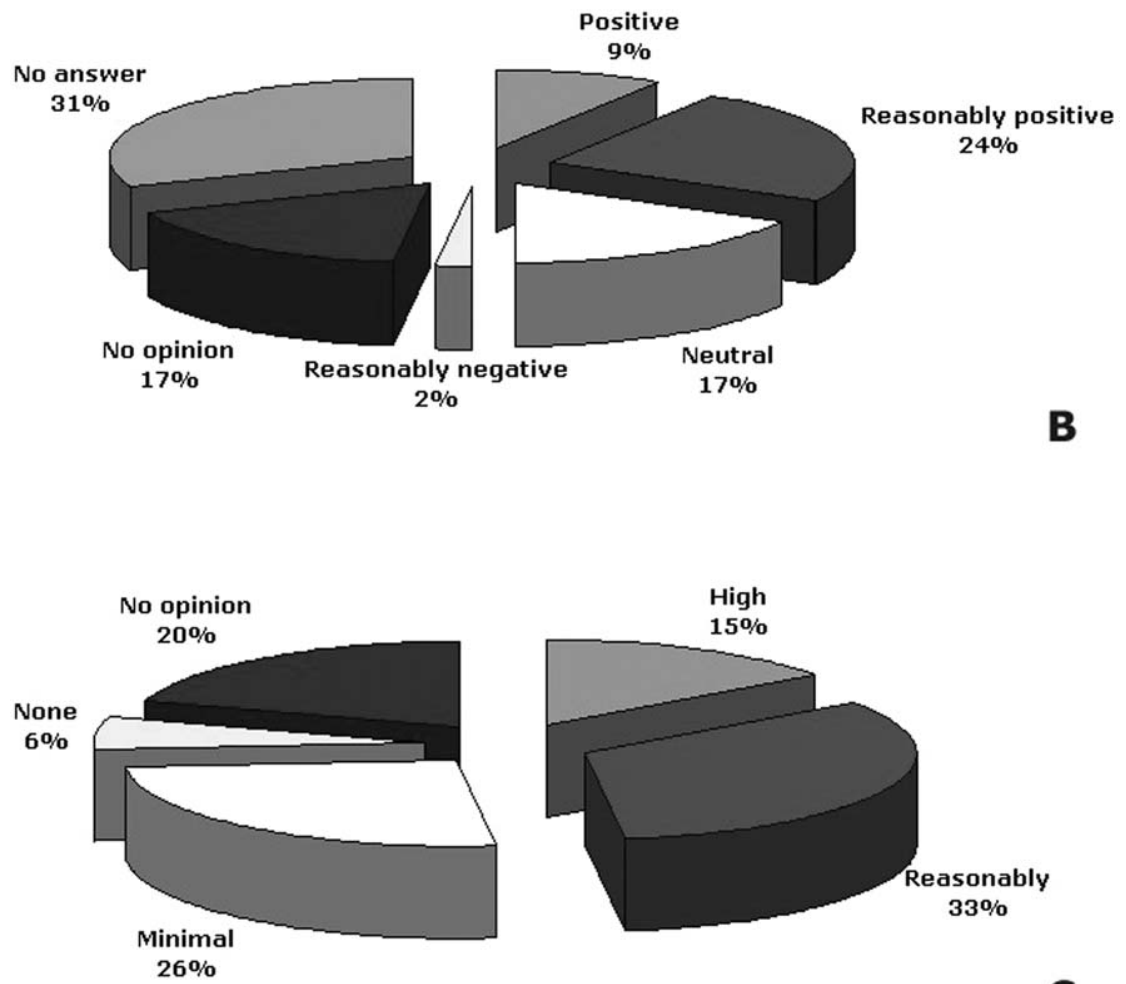

C

Figure 1 (A) Distinction between information and advertisement on websites of the pharmaceutical industry. (B) Value of information offered on websites of the pharmaceutical industry. (C) Estimated influence on doctor-patient relationship.

Like the indicated low impact of DTC communication, PASGs suggested that patients will not individually search the Internet for information about their diseases via industry websites (67\%). Also, they indicated that patients have little (24\%) or no (41\%) interest in contact with other patients via websites of the pharmaceutical industry.

\section{Patient-doctor relationship}

Answers on the question whether DTC communication had an impact on the patient-doctor relationship, shows mixed perceptions. Influence was estimated minimally $26 \%$, reasonably $33 \%$ to high $15 \%$ (20\% had no opinion) (Figure 1C). An influence on the prescribed medicines was expected by $39 \%$, while $30 \%$ expected a minor or no effect at all ( $28 \%$ had no opinion).

The question on the impact of DTC communication on the assertivity of the patient showed that $56 \%$ assumed an increase in assertivity by information of the pharmaceutical industry ( $22 \%$ had no opinion).

\section{Discussion}

In this pilot study in which e-mail was used to send the questionnaires, a high non-response was found. Especially the response from the industry was very low, which may have several reasons. Firstly, the participant might hesitate to reveal the company's marketing strategy. Secondly, the addressed person was not involved with this subject or thirdly because the receiver of the e-mail may not be interested enough to participate. This rather low number of responses of the pharmaceutical industry made the use of statistics not appropriate. There was no information available prior to this study to estimate the response of our questionnaire. The response on the e-mail questionnaires appeared to be low.

Some other annotations about this pilot study should also be made. The moment of sending the first e-mail was not optimal as summer holiday was about to begin. Furthermore, it was not necessary for responders to fill in all questions in order to send back the questionnaire. It is imaginable that an electronic 
version is usually answered more rapidly than a paper, which makes the risk to tick off a wrong answer or not answering higher.

PASGs instead of patients were asked their opinion about DTC communication because they are more accessible than large number of patients. However, their opinion might not be the same as that of the patients. Further research should clarify whether PASGs form a good representation of their members' opinion.

The attitude of PASGs towards DTC communication obtained via pharmaceutical websites is not clear. Half of the PASGs have not yet developed an opinion on DTC communication via the Internet or hesitate to declare an opinion. Of the responding PAGSs $74 \%$ expect that DTC has some kind of impact on the patientdoctor relationship; $56 \%$ assume an increase in assertivity.

Of the PASGs 15\% indicate that patients visiting an industry's website are able to distinguish between advertisement and information while $65 \%$ of the responders have no opinion on this subject. As this questionnaire addresses the individual PASG, it becomes clear that no real understanding and opinion has been developed at the level of individual PASGs yet. One of the reasons for this relative unawareness of the impact of DTC communication might be that the main users of prescription medicines are older patients. The Internet is a relative new medium and attracts mainly a younger public at the moment.

In the US, citizens are not obliged to participate in the health insurance system except the elderly and permanently disabled persons ${ }^{10}$. This is in contrast to the Dutch situation, where most medicines are refunded. DTC communication will be especially effective if the patient can easily influence the choice of medicines, e.g., if he has to pay for the medicines himself. The lack of direct influence of the Dutch patient on medicine prescription could play a role in explaining the current minor impact of DTC communication in the Netherlands. Therefore, discussions considering the legalization of DTC communication might be solely a political issue. Further research could investigate a possible relation between the impact of DTC communication and the health care system (open market versus governmental regulations).

\section{Conclusion}

The position of the pharmaceutical industry on DTC on prescription medicines via the Internet cannot be summarized conclusively due to their low response. However, there is an indication that the pharmaceutical industry is willing to invest in direct communication with the patients.

The current opinion of the PAGSs indicates that DTC communication via the Internet is not yet fully established in the Netherlands. The attitude of the PAGSs towards information obtained via websites is rather unclear.

Considering the different health care systems in the US and the Netherlands, the influence of DTC communication might be different in the Netherlands. The lack of direct influence of the Dutch patient on prescription medicines might play a role.

Further research is required to differentiate in the effect of DTC communication in the broad range of diseases and various types of medicines. The opinion of the pharmaceutical industry on their relation with their consumers needs more investigation.

\section{Acknowledgements}

We thank Dr A.H.P. Paes en Professor J.G.A.M. de Jong for their help in adjusting the questionnaires.

\section{References}

1 Holton MF. Direct-to-consumer marketing of prescription drugs creating consumer demand JAMA 1999; 281: 382-4.

2 Watson R. EC moves towards 'direct to consumer' advertising. BMJ 2001; 323: 184.

3 Anonymous. Retrieved from http://europa.eu.int/eur-lex/en/ $\mathrm{com} / \mathrm{pdf} / 2003 / \mathrm{com} 2003$ _0163en01.pdf on 18 July 2003.

4 Montagne M. Drug Advertising and Promotion: An Introduction. I Drug Issue 1992; 22: 195-203.

5 Raaijmakers JAM. Publieksreclame voor receptgeneesmiddelen... moet kunnen. [Advertising for prescription only medicines... it should be possible.] Pharmaceut W 2000; 135: 1481-82.

6 Hochhauser M. Which prescription for the illegible an unreadable DTC brief summary major surgery or euthanasia? Manager Care 2002; 10: 6-10.

7 Anonymous. FDA Division of Drug Marketing, Advertising and Communications. 1999 Attitudes and Behaviours Associated with DTC Promotion of Prescription Drugs: Main Survey Results. Retrieved from www.fda.gov/cder/ddmac/dtcindex.htm on 14 June 2002.

8 Spurgeon D. Doctors feel pressurized by direct to consumer advertising. BMJ 1999; 319: 1321.

9 Stergachis A, Maine LL, Brown L. The 2001 national pharmacy consumer survey. J Am Pharmaceut Assoc 2002; 42: 568-76.

10 Spaendonck van T, Douven R. Uitgavenontwikkelingen in de gezondheidszorg. CPB [Developments in the costs of public health.] Memorandum Centraal Planbureau, 24 July 2001. 